\title{
Clinical correlates of age at onset distribution in bipolar disorder: a comparison between diagnostic subgroups
}

\author{
Mirko Manchia ${ }^{1,2^{*}} \mathbb{0}$, Giuseppe Maina ${ }^{3}$, Bernardo Carpiniello ${ }^{1}$, Federica Pinna' ${ }^{1}$ Luca Steardo ${ }^{4}$, \\ Virginia D'Ambrosio ${ }^{3}$, Virginio Salvi ${ }^{3}$, Martin Alda ${ }^{5}$, Alfonso Tortorella ${ }^{4}$ and Umberto Albert ${ }^{6}$
}

\begin{abstract}
Background: Admixture analysis of age at onset (AAO) has helped delineating the clinical profile of early onset (EO) bipolar disorder (BD). However, there is scarce evidence comparing the distributional properties of AAO as well as the clinical features of EO BD type 1 (BD1) with EO BD type 2 (BD2). To this end, we studied 515 BD patients (224 BD1, 279 $\mathrm{BD} 2$, and $12 \mathrm{BD}$ not otherwise specified [NOS]) diagnosed according to DSM-IV-TR criteria.
\end{abstract}

Methods: AAO was defined as the first reliably diagnosed hypo/manic or depressive episode according to diagnostic criteria. We used normal distribution mixture analysis to identify subgroups of patients according to AAO. Models were chosen according to the Schwarz's Bayesian information criteria (BIC). Clinical correlates of EO were analysed using univariate tests and multivariate logistic regression models.

Results: A two normal components model best fitted the observed distribution of AAO in BD1 (BIC $=-1599.3), B D 2$ $(B I C=-2158.4)$, and in the whole sample $(B I C=-3854.9)$. A higher number of EO BD2 patients had a depression(hypo)mania-free interval (DMI) course, while a higher rate of (hypo)mania-depression-free interval (MDI) course was found in EO BD1. EO BD2 had also a higher rate of comorbidity with alcohol dependence compared to EO BD1. The latter finding was confirmed by multivariate logistic regression analysis.

Conclusions: In conclusion, both BD1 and BD2 had bimodal AAO distributions, but EO subgroups had a diagnosticspecific clinical delineation.

Keywords: Mood disorders, Diagnostic subtypes, Early onset, Retrospective study, Admixture analysis

\section{Background}

Bipolar disorder (BD) is a heritable psychiatric illness characterised by cyclic mood episodes of opposite polarity alternating with intervals of well-being (Goodwin and Jamison 2007). As in other psychiatric complex genetic diseases, the relatively high clinical heterogeneity of $\mathrm{BD}$ might have hindered the identification of molecular and clinical determinants of risk as well as of predictors of treatment outcome (Alda 2004). The magnitude of clinical heterogeneity might be reduced by studying subgroups of

\footnotetext{
*Correspondence: mirkomanchia@unica.it; Mirko.Manchia@dal.ca

1 Section of Psychiatry, Department of Medical Science and Public Health, University of Cagliari, Via Liguria, 13, 09127 Cagliari, Italy

Full list of author information is available at the end of the article
}

BD patients sharing specific clinical characteristics such as patterns of treatment response (Alda et al. 2005), mood incongruent psychosis (Goes et al. 2007), or early illness onset (Jamain et al. 2014). Indeed, the extensive analysis of age at onset (AAO) BD subgroups through admixture analysis has shown clinical (Bellivier et al. 2001; Lin et al. 2005; Manchia et al. 2008; Tozzi et al. 2011; Ortiz et al. 2011) and genetic (Etain et al. 2006; Severino et al. 2009; Etain et al. 2010; Belmonte et al. 2011) characteristics specific, particularly, to early onset (EO) BD.

As the vast majority of studies investigated $\mathrm{BD}$ type 1 (BD1) samples, it remains to be established, however, whether this clinical delineation of $\mathrm{EO}$ is present also in BD type 2 (BD2) patients. Furthermore, the distributional 
properties of AAO have never been investigated in samples exclusively composed of $\mathrm{BD} 2$ patients.

\section{Methods \\ Aims}

The primary aim of the present study was to test whether BD1 and BD2 differed in terms of AAO distributions. The secondary objective was to test whether EO had clinical characteristics specific for each diagnostic subgroup. To this end, we (i) studied the AAO distribution of each diagnostic subgroup with mixture modelling; (ii) compared the AAO distributions identified in BD1 and BD2; (iii) analyzed the pattern of associations of a set of demographic and clinical variables with EO in each diagnostic subgroup; and (iv) compared the clinical association patterns between $\mathrm{EO}$ BD1 and EO BD2.

\section{Patient population and assessment instruments}

Our sample consisted of 515 unrelated patients with BD. Two hundred and twenty-four were diagnosed with BD1, while 279 had a diagnosis of BD2, and 12 had a diagnosis BD not otherwise specified (NOS). All subjects were of Italian ancestry. Patients were recruited at the Anxiety and Mood Disorders Unit, University of Turin, Italy and at the Department of Psychiatry, University of Naples SUN, Napoli, Italy. Certified psychiatrists with at least 4 years of postgraduate clinical experience performed the clinical assessment of patients. All potential interviewers met prior to study beginning and underwent a common extensive training prior to conducting the assessments. They were trained in the use of a common semi-structured interview that was used to collect (a) socio-demographic data (age, gender, marital status, years of education, and occupational status); (b) diagnosis (current and lifetime), which were performed according to the Diagnostic and Statistical Manual of Mental Disorders (DSM)-IV-Text Revision (TR) criteria (American Psychiatric Association 2000) using the structured clinical interview for DSMIV-TR Axis I disorders (SCID-I/P) (First et al. 2002); (c) clinical data including AAO. In addition, a systematic review of patients' medical records helped clinicians to establish AAO and corroborate data concerning clinical characteristics of the disorder emerging from direct interview. Age at onset was defined as the first reliably diagnosed hypo/manic or depressive episode meeting the diagnostic criteria. External corroboration for AAO was obtained, whenever possible, by directly interviewing, with patient's consent, a first-degree family member or other significant individuals. For the purposes of the present study, we included only subjects for whom it was possible to establish AAO with complete agreement between the information provided by patients and their relatives. Age at interview was defined as the age at which subjects were first assessed by a clinician at each research centre.

In the early phase of the study, inter-rater reliability of the diagnosis of Axis I disorders with the SCID-I was ascertained. The inter-rater reliability was found to be good: Cohen kappa coefficient was 0.89 for the presence of any current or lifetime Axis I disorder.

\section{Data analysis}

We used Gaussian distribution mixture analysis to test whether we could identify subgroups of patients according to the AAO. We investigated a range of number of AAO groups (1-9). The choice of the mixture model that best fit the distribution of AAO was made according to the Schwarz's Bayesian information criteria (BIC). Specifically, the analysis performed with the "Mclust" (Fraley and Raftery 1999; Fraley et al. 2014) package implemented in R ( $\mathrm{R}$ Development Core Team 2008) indicates the best model as the one with the highest BIC among the fitted models (Fraley and Raftery 2007). This package estimates parameters of the model using an expectation-maximization (EM) algorithm. Cut-off points were derived using the Gaussian cumulative distribution function of estimated AAO mixture function and calculating each data point's probability of belonging to each class. Specifically, once the mixture model parameters were estimated, we calculated the posterior probability of any data point. The resulting probabilities were then compared in order to establish which class the data point belonged to. Gaussian mixture analysis (both number of components and parameters estimates) was also replicated and confirmed with the "Mixtools" R package (Benaglia et al. 2009). We used Kolmogorov-Smirnov $(\mathrm{K}-\mathrm{S})$ test to determine whether the Gaussian cumulative distribution function of estimated AAO mixture identified in BD1 patients was significantly different from the one identified in BD2 patients. Further, we used $\mathrm{K}-\mathrm{S}$ to test for differences in the AAO Gaussian cumulative distribution function of estimated AAO mixture between participating research centres. We tested the association of continuous and categorical clinical variables with AAO subgroups using univariate analysis ( $t$ test or $\chi^{2}$ test as appropriate). The independent variables tested included sex, age at interview, diagnosis, illness duration, presence of family history of any DSM-IV-TR psychiatric disorder, presence of family history of BD and any DSMIV-TR mood disorders, number of manic/hypomanic, depressive and mixed episodes, type of clinical course cycle [i.e. (hypo)mania-depression-free interval (MDI), depression-(hypo)mania-free interval (DMI), irregular cycling, continuous cycling, rapid cycling], presence of lifetime suicidal behaviour, lifetime comorbidity with 
substance (other than alcohol) dependence, and lifetime comorbidity with drug and/or alcohol dependence. Statistical significance was set at $\alpha=0.05$. Only clinical variables presenting a statistical significant association with an AAO subgroup $(p<0.05)$ of each diagnostic sample (BD1 and BD2) were entered into a backward stepwise multivariate binary logistic model to account for possible intercorrelations. All statistical analyses, except for mixture modelling, were performed with STATA/SE 12.0.

\section{Results}

\section{Age at onset distribution: Gaussian mixture analysis}

The BD1 sample (99 males and 125 females) had a mean age at interview $( \pm \mathrm{SD})$ of 47.2 years $( \pm 13.0)$ and a mean AAO of 26.7 years $( \pm 9.2)$. A two normal components model best fitted the observed distribution of AAO (BIC $=-1599.3$ ) (Fig. 1). Models with three and four components did not improve the fit (Table 1).

The EO component had a mean AAO of 22.6 years $( \pm 4.8)$, while the late onset $(\mathrm{LO})$ component had a mean AAO of 35.1 years $( \pm 10.1)$ comprising $67 \%$ and $33 \%$ of the population proportion, respectively. The cut-off point, derived by the Gaussian cumulative distribution function of the latter estimated AAO function, was at 32 years for BD1 (EO group <32 years; LO group $\geq 32$ years) with 169 patients in the EO group and 55 in the LO group.

The BD2 sample (114 males and 165 females) had a mean age at interview of 50.6 years $( \pm 14.8)$ and a mean AAO of 30.6 years $( \pm 12.7)$. The observed distribution of AAO was also best fitted by a two normal components model (BIC $=-2158.4$ ) (Fig. 2).

No improvement of the fit was observed with three and four components models (Table 1). The BD2 sample had an EO component with mean AAO of 20.9 years $( \pm 4.1)$ and a LO component with mean AAO of 38.2 years

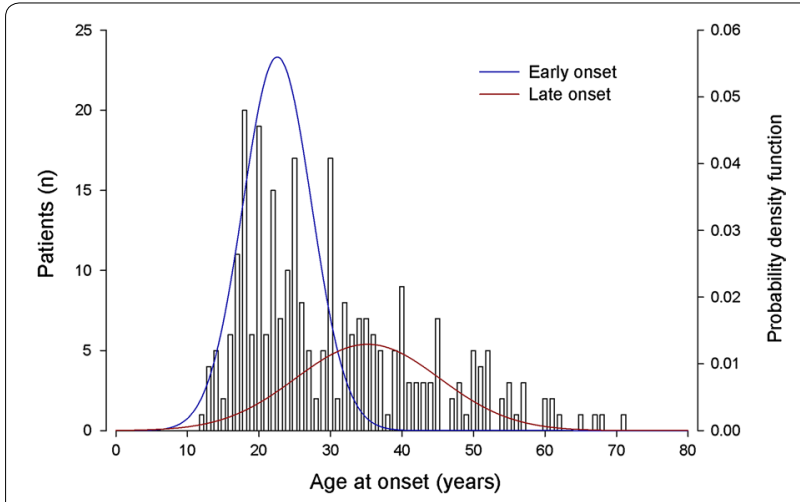

Fig. 1 Age at onset distribution in bipolar disorder type 1 patients $(N=224)$. Gaussian probability density function was derived by the estimated age at onset mixture function in bipolar disorder type 1 patients
Table 1 Age at onset distributions identified by Gaussian mixture analysis in bipolar disorder type 1 and type 2 and in the whole sample

\begin{tabular}{lllll}
\hline $\begin{array}{l}\text { Mixture } \\
\text { model }\end{array}$ & $\begin{array}{l}\text { Model compo- } \\
\text { nents }\end{array}$ & Mean SD & $\begin{array}{l}\text { Proportion } \\
(\%)\end{array}$
\end{tabular}

nents

(\%)

\begin{tabular}{|c|c|c|c|c|c|}
\hline \multicolumn{6}{|c|}{ Bipolar disorder type 1 sample $(n=224)$} \\
\hline M1 & -1637.7 & 1st component & 26.7 & 9.1 & 100.0 \\
\hline \multirow[t]{2}{*}{$M 2$} & -1599.4 & 1st component & 22.6 & 4.8 & 66.9 \\
\hline & & 2nd component & 35.1 & 10.1 & 33.1 \\
\hline \multirow[t]{3}{*}{ M3 } & -1611.0 & 1st component & 18.7 & 3.0 & 27.1 \\
\hline & & 2nd component & 25.6 & 4.4 & 45.7 \\
\hline & & 3rd component & 36.6 & 10.1 & 27.2 \\
\hline \multirow[t]{4}{*}{ M4 } & -1624.0 & 1st component & 17.9 & 2.6 & 23.1 \\
\hline & & 2nd component & 23.8 & 3.1 & 28.2 \\
\hline & & 3rd component & 28.8 & 5.9 & 33.4 \\
\hline & & 4th component & 40.9 & 9.9 & 15.3 \\
\hline \multicolumn{6}{|c|}{ Bipolar disorder type 2 sample $(n=279)$} \\
\hline M1 & -2218.4 & 1st component & 30.6 & 12.6 & 100.0 \\
\hline \multirow[t]{2}{*}{$M 2$} & -2158.4 & 1st component & 20.9 & 4.1 & 44.0 \\
\hline & & 2nd component & 38.2 & 11.8 & 56.0 \\
\hline \multirow[t]{3}{*}{ M3 } & -2163.2 & 1st component & 19.3 & 3.3 & 33.1 \\
\hline & & 2nd component & 28.4 & 6.1 & 33.7 \\
\hline & & 3rd component & 44.0 & 11.0 & 33.2 \\
\hline \multirow[t]{4}{*}{ M4 } & -2177.7 & 1st component & 17.6 & 2.5 & 22.8 \\
\hline & & 2nd component & 23.0 & 2.8 & 24.7 \\
\hline & & 3rd component & 32.2 & 4.4 & 22.7 \\
\hline & & 4th component & 45.6 & 10.3 & 29.8 \\
\hline \multicolumn{6}{|c|}{ Total bipolar disorder sample $(n=515)$} \\
\hline M1 & -3986.7 & 1st component & 29.0 & 11.5 & 100.0 \\
\hline \multirow[t]{2}{*}{$M 2$} & -3854.9 & 1st component & 21.9 & 4.6 & 55.0 \\
\hline & & 2nd component & 37.6 & 11.5 & 45.0 \\
\hline \multirow[t]{3}{*}{ M3 } & -3856.0 & 1st component & 19.2 & 3.2 & 28.9 \\
\hline & & 2nd component & 26.5 & 5.6 & 40.6 \\
\hline & & 3rd component & 41.5 & 11.2 & 30.6 \\
\hline \multirow[t]{4}{*}{ M4 } & -3868.3 & 1st component & 17.8 & 2.6 & 22.8 \\
\hline & & 2nd component & 23.3 & 3.1 & 28.0 \\
\hline & & 3rd component & 31.1 & 5.1 & 24.0 \\
\hline & & 4th component & 43.4 & 10.9 & 25.2 \\
\hline
\end{tabular}

Best fitting mixture models are typed in italics

$M$ model, BIC Bayesian information criteria, SD standard deviation

( \pm 11.8 ) with population proportions of $44 \%$ and $56 \%$, respectively. The cut-off point, derived by the Gaussian cumulative distribution function of this estimated AAO function, was at 28 years (EO group $<28$ years; LO group $\geq 28$ years). The EO group comprised 142 patients, while the LO group included 137 patients.

Kolmogorov-Smirnov test showed that the Gaussian cumulative distribution functions of estimated AAO mixture of BD1 and BD2 differed significantly $(D=0.18$, $p<0.00001$ ) (Fig. 3). Conversely, there was no significant difference between the Gaussian cumulative distributions 


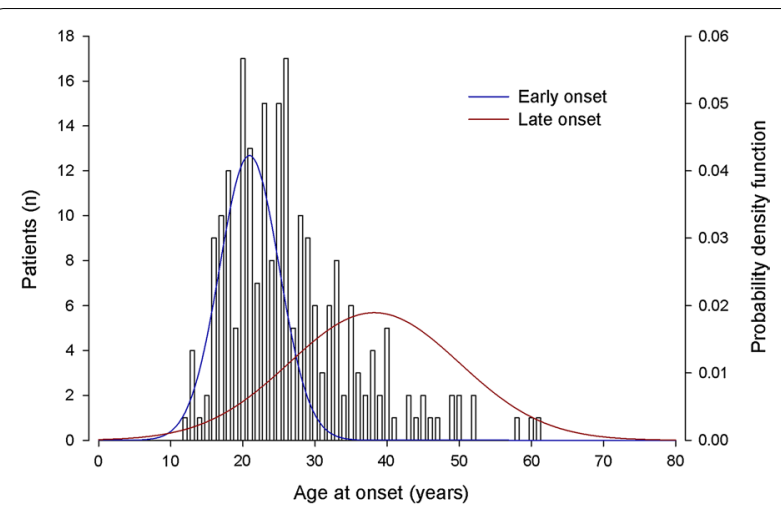

Fig. 2 Age at onset distribution in bipolar disorder type 2 patients $(N=279)$. Gaussian probability density function was derived by the estimated age at onset mixture function in bipolar disorder type 2 patients

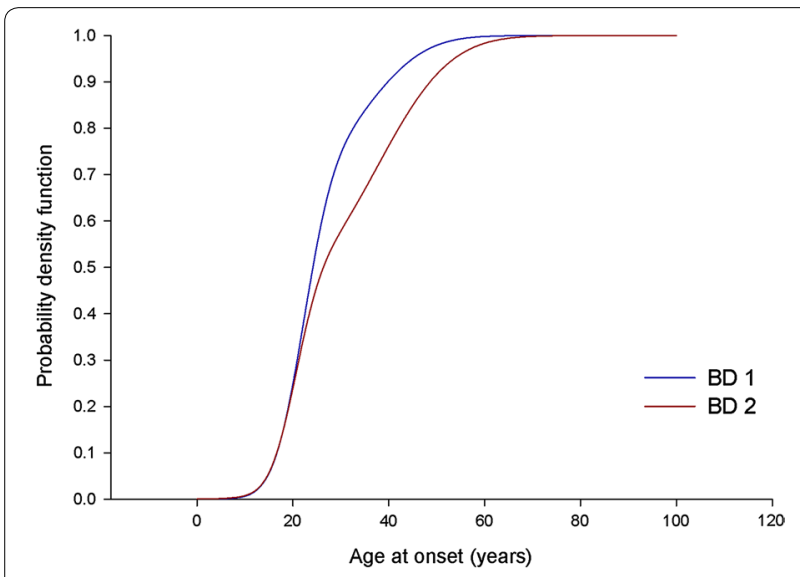

Fig. 3 Kolmogorov-Smirnov test between theoretical age at onset in bipolar disorder type 1 and type 2 patients. Probability density functions of the two estimated age at onset mixture function. BD 1 bipolar disorder type 1, BD2 bipolar disorder type 2

of estimated AAO mixture of the two participating research centres $(D=0.05, p=0.57)$. Finally, Gaussian mixture analysis confirmed a best fitting model of two normal components (detailed in Table 1) in the whole sample of 515 BD patients (216 males and 299 females), which included 12 subjects with BD NOS (Fig. 4). The cut-off point, derived by the Gaussian cumulative distribution function of this estimated AAO function, was at 30 years.

\section{Clinical correlates of early onset: patterns of association} in bipolar disorder type 1 and type 2 diagnostic subgroups As shown in Table 2 , a trend association $(p=0.05)$ was identified for the presence of family history of any DSMIV-TR mood disorder, with LO BD1 having a higher rate

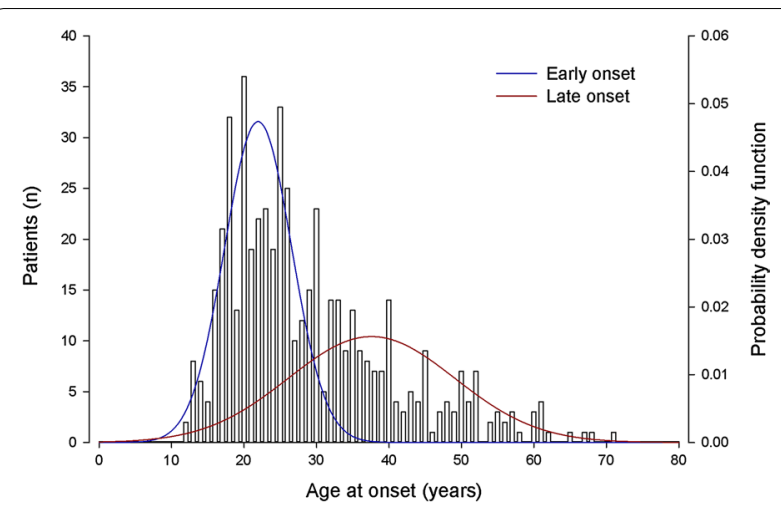

Fig. 4 Age at onset distribution in the whole sample of bipolar disorder patients $(N=515)$. Gaussian probability density function was derived by the estimated age at onset mixture function in the whole sample of bipolar disorder patients

than EO BD1. Early onset BD1 had a lower age at interview and a longer duration of illness than LO BD1.

Early onset BD2 had a higher rate of comorbidity with alcohol dependence, as well as a higher rate of family history of BD in EO BD2 compared to LO BD2 (Table 2). Further, they also had a lower age at interview and longer illness duration than LO BD2.

We then performed a multivariate logistic regression in BD2 confirming that family history of BD [odds ratio $(\mathrm{OR})=1.97$, 95\% confidence interval $(\mathrm{CI}) 1.04$ 3.73, $p=0.04$ ], comorbidity with alcohol dependence ( $\mathrm{OR}=3.2,95 \% \mathrm{CI} 1.21-8.54, p=0.02)$, and illness duration $(\mathrm{OR}=1.03,95 \%$ CI 1.008-1.048, $p=0.006)$ were associated with EO. As age at interview was used to calculate illness duration, and consequently significantly correlated with it $(r=-0.97)$, it was not included in the logistic regression model. Multivariate analysis was not performed in BD1 since no clinical variable was significantly associated with AAO subgroup in univariate test.

\section{Clinical correlates of early onset: comparison between bipolar disorder type 1 and type 2 diagnostic subgroups}

The mean AAO was significantly lower in BD1 compared to $\mathrm{BD} 2(26.7 \pm 9.2$ vs. $30.6 \pm 12.7 ; t=-3.8 ; p<0.0001)$. The results of the univariate analysis are shown in Table 3. Early onset BD1 patients were older than EO BD2. Conversely, EO BD2 had a higher rate of comorbidity with alcohol dependence. In addition, a higher number of EO BD2 presented with a DMI course, while a higher rate of MDI course was found in EO BD1. The multivariate binary logistic regression confirmed the association of comorbidity with alcohol dependence with EO BD2 (OR $=0.4,95 \%$ CI 0.18-0.90, $p=0.02)$. 
Table 2 Comparison of clinical correlates between early and late onset bipolar disorder type 1 and type 2 patients

\begin{tabular}{|c|c|c|c|c|}
\hline \multirow[t]{2}{*}{ Clinical variable } & \multicolumn{2}{|c|}{ Bipolar disorder type $1(N=224)$} & \multirow[t]{2}{*}{$x^{2}$ or $t$} & \multirow[t]{2}{*}{$P$} \\
\hline & Early onset $(N=169)$ & Late onset $(N=55)$ & & \\
\hline Female (\%) & $73(43.2)$ & $26(47.3)$ & 0.3 & 0.6 \\
\hline Age at interview, mean (SD) & $45.3(13.5)$ & $53.1(8.8)$ & 4.0 & $<0.0001$ \\
\hline Presence of family history of any psychiatric disorder (\%) & $99(58.9)$ & $37(67.3)$ & 1.2 & 0.3 \\
\hline Presence of family history of mood disorder (\%) ${ }^{\mathrm{b}}$ & $85(52.5)$ & $34(68.0)$ & 3.7 & 0.05 \\
\hline Presence of family history of bipolar disorder (\%) ${ }^{c}$ & $31(18.5)$ & $13(23.6)$ & 0.7 & 0.4 \\
\hline Number of manic/hypomanic episodes, mean (SD) ${ }^{d}$ & $4.4(5.1)$ & $3.6(3.2)$ & -1.1 & 0.3 \\
\hline Number of depressive episodes, mean (SD) ${ }^{e}$ & $5.5(4.7)$ & $4.8(4.4)$ & -0.9 & 0.3 \\
\hline Number of mixed episodes, mean $(S D)^{f}$ & $0.6(1.6)$ & $0.8(1.3)$ & 0.8 & 0.4 \\
\hline Illness duration, mean (SD) & $22.5(12.8)$ & $13.5(8.6)$ & -4.9 & $<0.0001$ \\
\hline Lifetime comorbidity with substance dependence (\%) & $15(8.9)$ & $4(7.3)$ & 0.1 & 0.7 \\
\hline Lifetime comorbidity with alcohol dependence (\%) & $10(5.9)$ & $1(1.8)$ & 1.5 & 0.2 \\
\hline Presence of suicidal behaviour (\%) & $47(28)$ & $11(20)$ & 1.4 & 0.2 \\
\hline \multicolumn{5}{|l|}{ Type of clinical course cycle } \\
\hline MDI (\%) & $75(44.4)$ & $22(40.0)$ & 3.7 & 0.45 \\
\hline DMI (\%) & $20(11.8)$ & $11(20.0)$ & & \\
\hline Irregular cycling (\%) & $65(38.5)$ & $21(38.2)$ & & \\
\hline Continuous cycling (\%) & $5(3.0)$ & $1(1.8)$ & & \\
\hline Rapid cycling (\%) & $4(2.4)$ & $0(0.0)$ & & \\
\hline \multirow[t]{2}{*}{ Clinical variable } & \multicolumn{2}{|c|}{ Bipolar disorder type $2(N=279)$} & \multirow[t]{2}{*}{$x^{2}$ or $t$} & \multirow[t]{2}{*}{$p$} \\
\hline & Early onset $(N=142)$ & Late onset $(N=137)$ & & \\
\hline Female (\%) & $85(59.9)$ & $80(58.4)$ & 0.06 & 0.8 \\
\hline Age at interview, mean (SD) & $42.8(14.4)$ & $58.7(10.1)$ & 10.6 & $<0.0001$ \\
\hline Presence of family history of any psychiatric disorder (\%) & $93(65.5)$ & $87(63.5)$ & 0.1 & 0.7 \\
\hline Presence of family history of mood disorder $(\%)^{b}$ & $84(60.0)$ & $79(58.5)$ & 0.06 & 0.8 \\
\hline Presence of family history of bipolar disorder (\%) & $36(25.4)$ & $19(13.9)$ & 5.8 & 0.02 \\
\hline Number of manic/hypomanic episodes, mean (SD) ${ }^{d}$ & $4.3(4.2)$ & $3.7(4.3)$ & -1.1 & 0.3 \\
\hline Number of depressive episodes, mean (SD) $)^{e}$ & $6.0(5.6)$ & $5.4(5.3)$ & -1.0 & 0.3 \\
\hline Illness duration, mean (SD) ${ }^{g}$ & $22.0(13.7)$ & $17.8(11.2)$ & -2.8 & 0.005 \\
\hline Lifetime comorbidity with substance dependence (\%) & $8(5.6)$ & $6(4.4)$ & 0.2 & 0.6 \\
\hline Lifetime comorbidity with alcohol dependence (\%) & $19(13.4)$ & $6(4.4)$ & 6.9 & 0.01 \\
\hline Presence of suicidal behaviour (\%) & $45(31.7)$ & $29(21.2)$ & 3.9 & 0.06 \\
\hline \multicolumn{5}{|l|}{ Type of clinical course cycle } \\
\hline MDI (\%) & $51(35.9)$ & $45(32.8)$ & 3.9 & 0.4 \\
\hline DMI (\%) & $31(21.8)$ & $35(25.5)$ & & \\
\hline Irregular cycling (\%) & $58(40.8)$ & $52(38.0)$ & & \\
\hline Continuous cycling (\%) & $2(1.4)$ & $2(1.5)$ & & \\
\hline Rapid cycling (\%) & $0(0.0)$ & $3(2.2)$ & & \\
\hline
\end{tabular}

Significant values are typed in italics

$M D I$ (hypo)mania-depression-free interval, DMI depression-(hypo)mania-free interval, SD Standard Deviation, $p \mathrm{p}$ value

a BD1: missing data for 1 patient

b BD1: missing data for 12 patients, BD2: missing data for 4 patients

c BD1: missing data for 1 patient

d BD1: missing data for 1 patient, BD2: missing data for 4 patients

e BD1: missing data for 1 patient, BD2: missing data for 4 patients

f BD1: missing data for 1 patient, BD2: missing data for 3 patients

$g$ BD2: missing data for 2 patients 
Table 3 Comparison of clinical correlates between early onset bipolar disorder type 1 and type 2 patients

\begin{tabular}{|c|c|c|c|c|}
\hline \multirow[t]{2}{*}{ Clinical variable } & \multicolumn{2}{|l|}{ Early onset diagnostic subgroup } & \multirow[t]{2}{*}{$x^{2}$ or $t$} & \multirow[t]{2}{*}{$P$} \\
\hline & Bipolar disorder type $1(N=169)$ & Bipolar disorder type $2(N=142)$ & & \\
\hline Female (\%) & $96(56.8)$ & $85(59.9)$ & 0.3 & 0.6 \\
\hline Age at interview, mean (SD) & $45.3(13.5)$ & $42.8(14.4)$ & 1.5 & 0.01 \\
\hline Presence of family history of any psychiatric disorder (\%) & $99(58.9)$ & $93(65.5)$ & 1.4 & 0.2 \\
\hline Presence of family history of mood disorder (\%) & $85(52.5)$ & $84(60.0)$ & 1.7 & 0.2 \\
\hline Presence of family history of bipolar disorder (\%) & $31(18.5)$ & $36(25.4)$ & 2.2 & 0.1 \\
\hline Number of manic/hypomanic episodes, mean (SD) & $4.4(5.1)$ & $4.3(4.2)$ & 0.2 & 0.8 \\
\hline Number of depressive episodes, mean (SD) & $5.5(4.7)$ & $6.0(5.6)$ & -0.8 & 0.4 \\
\hline Illness duration, mean (SD) & $22.5(12.8)$ & $21.9(13.7)$ & 0.4 & 0.7 \\
\hline Lifetime comorbidity with substance dependence (\%) & $15(8.9)$ & $8(5.6)$ & 1.2 & 0.3 \\
\hline Lifetime comorbidity with alcohol dependence (\%) & $10(5.9)$ & $19(13.4)$ & 5.1 & 0.02 \\
\hline Presence of suicidal behaviour (\%) & $47(28.0)$ & $45(31.7)$ & 0.5 & 0.5 \\
\hline \multicolumn{5}{|l|}{ Type of clinical course cycle } \\
\hline MDI (\%) & $75(44.4)$ & $51(35.9)$ & 10.4 & 0.035 \\
\hline DMI (\%) & $20(11.8)$ & $31(21.8)$ & & \\
\hline Irregular cycling (\%) & $65(38.5)$ & $58(40.8)$ & & \\
\hline Continuous cycling (\%) & $5(3.0)$ & $2(1.4)$ & & \\
\hline Rapid cycling (\%) & $4(2.4)$ & $0(0.0)$ & & \\
\hline
\end{tabular}

Significant values are typed in italics

MDI (hypo)mania-depression-free interval, DMI depression-(hypo)mania-free interval, SD Standard Deviation, $p \mathrm{p}$ value

\section{Discussion}

The present study highlighted that a two normal component model in BD1 as well as in BD2 diagnostic subgroup best described the distribution of AAO. This finding was not reflected, however, in similar distributional properties of AAO, as well as in comparable pattern of association with clinical variables between the two diagnostic subgroups. In fact, our study found that EO BD2 patients had a higher rate of alcohol dependence compared to both LO BD2 and EO BD1. Further, EO BD2 patients had more frequently a DMI type of clinical course, while the MDI type was more frequently associated with EO BD1. Finally, EO BD2 showed a higher familial load for BD compared to LO BD.

The bimodal AAO distribution found in both $\mathrm{BD}$ diagnostic subgroups and in the whole sample of 515 patients is consistent with some studies (Ortiz et al. 2011; Kennedy et al. 2005; Javaid et al. 2011). Conversely, the majority of studies on mixture analysis of AAO showed a trimodal distribution in samples comprising mainly BD1 patients (Bellivier et al. 2001, 2003; Lin et al. 2006; Severino et al. 2009; Hamshere et al. 2009; Tozzi et al. 2011; Bellivier et al. 2014; Golmard et al. 2015). Of note, a recent study showed that bimodal and trimodal distribution fit equally well the AAO of BD (GrigoroiuSerbanescu et al. 2014). Further research is needed to determine which distribution (bi- or tri-modal) better describes $\mathrm{AAO}$ in $\mathrm{BD}$ and which is (or which are) the best cut-off(s) before investigating clinical correlates and genetic differences between subgroups based on AAO. In fact, thresholds between subgroups found in different studies differed [e.g. thresholds between the intermediate and late AAO subgroups differed from 25 in one study (Tozzi et al. 2011) to 40 years in another (Hamshere et al. 2009)] as well as percentages of patients in each AAO subgroups [e.g. percentages of patients attributed to the early onset subgroup varied between $21.4 \%$ (Bellivier et al. 2001) and 79.7\% (Lin et al. 2006)]. Discrepancies in the identified AAO distributions, cut-off points, and proportions of patients in each AAO subgroups may depend on diverse assessment methods, recall bias, study design (Montlahuc et al. 2016), and differences in characteristics of samples studied, including geographic location (Post et al. 2008; Bellivier et al. 2014) and birth cohort (Bauer et al. 2015; Golmard et al. 2015). Concerning study design, Montlahuc et al. (2016) tested whether cross-sectional designs (which cause right truncation), unreliable diagnosis for individuals younger than 10 years old (which causes left truncation), and the selection criterion used for admixture analysis impacted the number of identified AAO subgroups. Importantly, a combination of left and right truncation, which is common in previously published studies of AAO admixture analysis, appeared to significantly influence the number of AAO subgroups detected (Montlahuc et al. 2016). Geographical location appears also to impact on AAO admixture 
analysis findings. Bellivier et al. (2014) found significant differences in the theoretical AAO functions between USA and European BD samples, mainly led by the higher proportion of patients in the EO subgroup and the lower mean AAO in the USA sample. Finally, birth cohort effect might also influence the estimation of AAO subgroups parameters. In this regard, Golmard et al. (2015) found that the proportion of EO cases increased substantially among BD cases born after 1960 compared to those born before the same year.

Several other findings deserve a comment. In our sample, BD type 1 patients had an earlier mean AAO (26.7 years) than BD2 patients (30.6 years), in agreement with existing data showing that BD1 first manifest their symptoms at an earlier age (Merikangas et al. 2011). In keeping, admixture analyses indicated a larger proportion of EO cases among BD1 patients (67\%) compared to $\mathrm{BD} 2$ patients $(44 \%)$. As a consequence, the EO BD1 group had a later mean AAO (22.6 years), compared to EO BD2 patients (20.9 years), reflecting in a higher AAO cut-off point (32 years for BD1 and 28 years for BD2).

These distributional properties of AAO distinguishing $\mathrm{EO} \mathrm{BD} 1$ from $\mathrm{EO} \mathrm{BD} 2$ resulted also in diverse patterns of clinical correlates of EO. Indeed, EO BD2 patients had a higher rate of alcohol dependence compared to both LO BD2 and EO BD1. Similarly, previous studies investigating clinical correlates of AAO subgroups found that EO BD patients have higher rates of alcohol abuse (Javaid et al. 2011; Lin et al. 2006). However, these studies either analysed only BD1 individuals (Lin et al. 2006) or did not specify the diagnostic stratification (Javaid et al. 2011). Interestingly, a recent study from Propper et al. (2015) did not find differences in rates of alcohol abuse among AAO subgroups in a sample with a $\mathrm{BD} 1: \mathrm{BD} 2$ ratio of 2:1.

Early onset BD2 patients had more frequently a DMI type of clinical course, while the MDI type was more frequently associated with EO BD1. Although not directly comparable with our study, the findings reported by Perlis et al. (2004) and Propper et al. (2015) in their samples relatively balanced in terms of $\mathrm{BD} 1: \mathrm{BD} 2$ ratio, indicated that very $\mathrm{EO}$ and $\mathrm{EO} \mathrm{BD}$ patients have more frequently onset episodes of depressive polarity compared to later onset subgroups. In addition, our findings were consistent with the work of Koukopoulos et al. (2013), which found that patients with a DMI illness course are more likely to be BD2, while MDI illness course is overrepresented in BD1 patients.

Differently from what reported in the literature, our study failed to confirm in the BD1 subgroup the wellestablished association of EO BD with a higher familial load for BD and for mood disorders in general, as well as with higher rates of suicidal behaviour (Geoffroy et al.
2013). On the contrary, EO BD2 showed a higher familial load for $\mathrm{BD}(p=0.02)$ compared to LO BD. Finally, although not statistically significant, EO BD2 showed higher rates of family history for BD as well as for mood disorders, compared to EO BD1. Similarly, Baek et al. (2011) found higher rates of major depression, but not of $\mathrm{BD}$, in $\mathrm{BD} 2$ patients compared to BD1, although their sample was not stratified according to AAO. Further, another recent study showed that both BD1 and BD2 presented a similar familial load for mood disorders (Dell'Osso et al. 2016)

There is compelling evidence that EO BD patients appear also to be more frequently associated with rapid cycling, drug abuse, higher rates of obsessive-compulsive disorder, and possibly for psychotic features, and panic disorder (Geoffroy et al. 2013). Although our study did not test for association most of these clinical correlates, there were no statistically significant associations with EO for drug dependence. Of note, most of this evidence is derived from BD1 samples as there is a lack of data on the analysis of AAO in BD2, while only a few studies investigated mixed samples with both BD1 and BD2 patients (Perlis et al. 2004; Severino et al. 2009; Tozzi et al. 2011; Ortiz et al. 2011; Propper et al. 2015).

Our results should be interpreted in the context of some limitations. The retrospective assessment of AAO might have been subject to recall bias. However, data were gathered through direct interview of the patients as well as with systematic review of medical charts decreasing the probability of a systematic bias in the assessment of AAO. An additional limitation is the lack of a systematic approach in collecting family history data, which might have influenced the assessment of familial load in our sample. Moreover, external corroboration for AAO was obtained, whenever possible, by directly interviewing a first-degree family member or other significant individuals. Further, our samples of BD1 and BD2 patients might not have had an adequate statistical power to detect association signals of small to moderate magnitude. Finally, our study did not consider birth cohort effect in our analysis.

\section{Conclusions}

To our knowledge, this is the first study specifically aimed at comparing clinical correlates of EO between BD1 and BD2 patients' populations using admixture analysis. Our work found that, beside diverse distributional properties of AAO, BD1 and BD2 EO subgroups differed also in their clinical characteristics. Of note, our study identified a subgroup of EO BD2 with an AAO even earlier than in the EO BD1 subgroup, characterised by a higher genetic load (i.e. higher familial load for BD) and at greater risk of developing alcohol dependence. Should our findings 
be replicated in other studies directly comparing AAO BD1 subgroups with AAO BD2 subgroups, future work will be able to focus also on the differences in the genetic and biological makeup, possibly facilitating the search for reliable disease biomarkers.

\section{Abbreviations}

BD: bipolar disorder; BD1: BD type 1; BD2: BD type 2; AAO: age at onset; EO: early onset; LO: late onset; NOS: not otherwise specified; BIC: Schwarz's Bayesian information criteria; DMl: depression-(hypo)mania-free interval; MDI: (hypo)mania-depression-free interval; SCID-I/P: structured clinical interview for DSM-IV-TR Axis I disorders; KS: Kolmogorov-Smirnov.

\section{Authors' contributions}

MM performed the statistical analysis and wrote the initial draft of the manuscript. GM designed the study, oversaw data analysis, and critically revised the manuscript. BC contributed to the study design, and critically revised the manuscript. FP contributed to the study design, and critically revised the manuscript. LS, VDA, and VS collected clinical data, and critically revised the manuscript. MA oversaw data analysis, and critically revised the manuscript. AT designed the study, oversaw data analysis, and performed some of the writing. UA designed the study, oversaw data analysis, and performed some of the writing. All authors read and approved the final manuscript.

\section{Author details \\ 1 Section of Psychiatry, Department of Medical Science and Public Health, University of Cagliari, Via Liguria, 13, 09127 Cagliari, Italy. ${ }^{2}$ Department of Pharmacology, Dalhousie University, Halifax, NS, Canada. ${ }^{3}$ Department of Mental Health, "San Luigi-Gonzaga" Hospital, University of Turin, Orbas- sano, Italy. ${ }^{4}$ Department of Psychiatry, University of Naples SUN, Naples, Italy. ${ }^{5}$ Department of Psychiatry, Dalhousie University, Halifax, NS, Canada. ${ }^{6}$ Rita Levi Montalcini Department of Neuroscience, Anxiety and Mood Disorders Unit, University of Turin, Turin, Italy.}

\section{Acknowledgements}

None.

\section{Competing interests}

The authors have no other relevant affiliations or financial involvement with any organisation or entity with a financial interest in or financial conflict with the subject matter or materials discussed in the manuscript.

\section{Ethics}

After a detailed description of the study procedures, informed written consent to participate in the study was obtained from all patients. The local Ethical Committees from each participating centre approved the study.

\section{Funding}

This research did not receive any specific grant from funding agencies in the public, commercial, or not-for-profit sectors.

\section{Publisher's Note}

Springer Nature remains neutral with regard to jurisdictional claims in published maps and institutional affiliations.

Received: 6 January 2017 Accepted: 26 April 2017

Published online: 21 August 2017

\section{References}

Alda M. The phenotypic spectra of bipolar disorder. Eur Neuropsychopharmacol. 2004;14(Suppl 2):S94-9.

Alda M, Grof P, Rouleau GA, Turecki G, Young LT. Investigating responders to lithium prophylaxis as a strategy for mapping susceptibility genes for bipolar disorder. Prog Neuropsychopharmacol Biol Psychiatry. 2005;29(6):1038-45
American Psychiatric Association. Diagnostic and statistical manual of mental disorders (4th ed. text rev.). 1st ed. Washington, DC: American Psychiatric Association; 2000

Baek JH, Park DY, Choi J, Kim JS, Choi JS, Ha K, et al. Differences between bipolar I and bipolar II disorders in clinical features, comorbidity, and family history. J Affect Disord. 2011;131(1-3):59-67.

Bauer M, Glenn T, Alda M, Andreassen OA, Angelopoulos E, Ardau R, et al. Influence of birth cohort on age of onset cluster analysis in bipolar I disorder. Eur Psychiatry. 2015;30(1):99-105.

Bellivier F, Etain B, Malafosse A, Henry C, Kahn JP, Elgrabli-Wajsbrot O, et al. Age at onset in bipolar I affective disorder in the USA and Europe. World J Biol Psychiatry. 2014;15(5):369-76.

Bellivier F, Golmard JL, Henry C, Leboyer M, Schurhoff F. Admixture analysis of age at onset in bipolar I affective disorder. Arch Gen Psychiatry. 2001;58(5):510-2.

Bellivier F, Golmard JL, Rietschel M, Schulze TG, Malafosse A, Preisig M, et al Age at onset in bipolar I affective disorder: further evidence for three subgroups. Am J Psychiatry. 2003;160(5):999-1001.

Belmonte MP, Pirooznia M, Goes FS, Seifuddin F, Steele J, Lee PH, et al. Genome-wide association analysis of age at onset and psychotic symptoms in bipolar disorder. Am J Med Genet B Neuropsychiatr Genet 2011;156B(3):370-8

Benaglia T, Chauveau D, Hunter DR, Young D. mixtools: an R package for analyzing finite mixture models. J Stat Softw. 2009;32(6):1-29.

Dell'Osso B, Dobrea C, Cremaschi L, Buoli M, Miller S, Ketter TA et al. Italian bipolar II vs I patients have better individual functioning, in spite of overall similar illness severity. CNS Spectr 2016;1-8.

Etain B, Dumaine A, Mathieu F, Chevalier F, Henry C, Kahn JP, et al. A SNAP25 promoter variant is associated with early-onset bipolar disorder and a high expression level in brain. Mol Psychiatry. 2010;15(7):748-55.

Etain B, Mathieu F, Rietschel M, Maier W, Albus M, McKeon P, et al. Genomewide scan for genes involved in bipolar affective disorder in 70 European families ascertained through a bipolar type I early-onset proband: supportive evidence for linkage at 3p14. Mol Psychiatry. 2006;11(7):685-94.

First MB, Spitzer RL, Gibbon M, Williams JBW. Structured clinical interview for DSM-IV-TR Axis I disorders, Research Version, Patient Edition. (SCID-I/P). New York: Biometrics Research, New York State Psychiatric Institute;2002.

Fraley C, Raftery A. MCLUST: software for model-based cluster analysis. J Classif. 1999;16(2):297-306.

Fraley C, Raftery A, Scrucca L. Normal mixture modeling for model-based clustering, classification, and density estimation. R 2014;(4.3).

Fraley C, Raftery AE. Model-based methods of classification: using the mclust software in chemometrics. J Stat Softw. 2007;18(6):1-13.

Geoffroy PA, Etain B, Scott J, Henry C, Jamain S, Leboyer M, et al. Reconsideration of bipolar disorder as a developmental disorder: importance of the time of onset. J Physiol Paris. 2013;107(4):278-85.

Goes FS, Zandi PP, Miao K, McMahon FJ, Steele J, Willour VL, et al. Moodincongruent psychotic features in bipolar disorder: familial aggregation and suggestive linkage to 2p11-q14 and 13q21-33. Am J Psychiatry. 2007;164(2):236-47.

Golmard JL, Scott J, Etain B, Preisig M, Aubry JM, Henry C, et al. Using admixture analysis to examine birth-cohort effects on age at onset of bipolar disorder. Acta Psychiatr Scand. 2015:133(3):205-13.

Goodwin FK, Jamison KR. Course and outcome. In: Goodwin FK, Jamison KR, editors. Manic-depressive illness. New York: Oxford University Press; 2007. p. 119-54.

Grigoroiu-Serbanescu M, Rietschel M, Hauser J, Czerski PM, Herms S, Sun X, et al. Commingling analysis of age-of-onset in bipolar I disorder and the morbid risk for major psychoses in first degree relatives of bipolar I probands. J Affect Disord. 2014;168:197-204.

Hamshere ML, Gordon-Smith K, Forty L, Jones L, Caesar S, Fraser C, et al. Ageat-onset in bipolar-I disorder: mixture analysis of 1369 cases identifies three distinct clinical sub-groups. J Affect Disord. 2009;116(1-2):23-9.

Jamain S, Cichon S, Etain B, Muhleisen TW, Georgi A, Zidane N, et al. Common and rare variant analysis in early-onset bipolar disorder vulnerability. PLoS ONE 2014:9(8):e104326.

Javaid N, Kennedy JL, De Luca V. Ethnicity and age at onset in bipolar spectrum disorders. CNS Spectr. 2011;16(6):127-34.

Kennedy N, Everitt B, Boydell J, VAN Os J, Jones PB, Murray RM. Incidence and distribution of first-episode mania by age: results from a 35-year study. Psychol Med. 2005;35(6):855-63. 
Koukopoulos A, Reginaldi D, Tondo L, Visioli C, Baldessarini RJ. Course sequences in bipolar disorder: depressions preceding or following manias or hypomanias. J Affect Disord. 2013;151(1):105-10.

Lin PI, McInnis MG, Potash JB, Willour V, MacKinnon DF, Depaulo JR, et al. Clinical correlates and familial aggregation of age at onset in bipolar disorder. Am J Psychiatry. 2006;163(2):240-6.

Lin PI, McInnis MG, Potash JB, Willour VL, MacKinnon DF, Miao K, et al. Assessment of the effect of age at onset on linkage to bipolar disorder: evidence on chromosomes 18p and 21q. Am J Hum Genet. 2005;77(4):545-55.

Manchia M, Lampus S, Chillotti C, Sardu C, Ardau R, Severino G, et al. Age at onset in Sardinian bipolar I patients: evidence for three subgroups. Bipolar Disord. 2008;10(3):443-6.

Merikangas KR, Jin R, He JP, Kessler RC, Lee S, Sampson NA, et al. Prevalence and correlates of bipolar spectrum disorder in the world mental health survey initiative. Arch Gen Psychiatry. 2011;68(3):241-51.

Montlahuc C, Curis E, Jonas SF, Bellivier F, Chevret S. Age-at-onset subsets of bipolar I disorders: a critical insight into admixture analyses. Int J Methods Psychiatr Res. 2016;1-8.

Ortiz A, Bradler K, Slaney C, Garnham J, Ruzickova M, O'Donovan C, et al. An admixture analysis of the age at index episodes in bipolar disorder. Psychiatry Res. 2011;188(1):34-9.
Perlis RH, Miyahara S, Marangell LB, Wisniewski SR, Ostacher M, DelBello MP, et al. Long-term implications of early onset in bipolar disorder: data from the first 1000 participants in the systematic treatment enhancement program for bipolar disorder (STEP-BD). Biol Psychiatry. 2004;55(9):875-81.

Post RM, Luckenbaugh DA, Leverich GS, Altshuler LL, Frye MA, Suppes T, et al. Incidence of childhood-onset bipolar illness in the USA and Europe. Br J Psychiatry. 2008;192(2):150-1.

Propper L, Ortiz A, Slaney C, Garnham J, Ruzickova M, Calkin CV, et al. Earlyonset and very-early-onset bipolar disorder: distinct or similar clinical conditions? Bipolar Disord. 2015;17(8):814-20.

R Development Core Team. R: a language and environment for statistical computing. R 2008

Severino G, Manchia M, Contu P, Squassina A, Lampus S, Ardau R, et al. Association study in a Sardinian sample between bipolar disorder and the nuclear receptor REV-ERBalpha gene, a critical component of the circadian clock system. Bipolar Disord. 2009;11(2):215-20.

Tozzi F, Manchia M, Galwey NW, Severino G, Del Zompo M, Day R, et al. Admixture analysis of age at onset in bipolar disorder. Psychiatry Res. 2011;185(1-2):27-32.

\section{Submit your manuscript to a SpringerOpen ${ }^{\circ}$ journal and benefit from:}

- Convenient online submission

- Rigorous peer review

- Immediate publication on acceptance

- Open access: articles freely available online

- High visibility within the field

- Retaining the copyright to your article 